\title{
Avistamientos de Orcinus orca (Linnaeus, 1758) (Cetartiodactyla: Odontoceti: Delphinidae) en el Pacífico costarricense (1990-2020)
}

\section{Sightings of Orcinus orca (Linnaeus, 1758) (Cetartiodactyla: Odontoceti: Delphinidae) in the Costa Rican Pacific (1990-2020)}

\author{
César Castro-Azofeifa ${ }^{1}$
}

\begin{abstract}
RESUMEN
Las orcas son cetáceos poco estudiados en el trópico, y sus desplazamientos por aguas costarricenses han sido escasamente abordados. Son animales de gran importancia ecológica debido a su rol como depredadores top, y documentar su presencia en el país puede ser de gran importancia científica y social, pues para las comunidades costeras esta información podría representar una gran oportunidad desde el punto de vista ecoturístico. El objetivo de esta investigación fue sintetizar y sistematizar los reportes existentes, referente a las orcas en el país, mediante publicaciones científicas, libros e información derivada de informes de ciudadanos. Se tomó en cuenta la información recabada hasta el 31 de diciembre de 2020. Se dividieron los avistamientos y el número de orcas en el Pacífico costarricense regionalmente: 17 avistamientos y 56 individuos para el Pacífico Sur, 7 avistamientos y 20 individuos para el Pacífico Norte, 3 avistamientos y 19 individuos en el Pacífico Central y 4 avistamientos y 10 individuos para la Isla del Coco. Se contabilizaron 31 avistamientos y 105 individuos en total. No se encontraron diferencias estadísticamente significativas sobre la ocurrencia de orcas entre las zonas, aunque hay una mayor cantidad de reportes en el Pacífico Sur, en especial, durante setiembre. Estudiar la presencia de las orcas en el país, que involucren procesos de educación ambiental y ciencia ciudadana, puede proveer importantes herramientas para la formulación de políticas públicas que permitan una conservación efectiva de los cetáceos y ofrecer mayores oportunidades de crecimiento para la industria ecoturística en las costas.
\end{abstract}

Palabras clave: ballenas asesinas tropicales, ciencia ciudadana, desplazamiento de cetáceos, ecología de mamíferos marinos tropicales, hábitos predatorios

1 Estudiante de grado, Escuela de Biología, Universidad de Costa Rica; 11501-2060 San Pedro, San José, Costa Rica; cesar.castroazofeifa@ucr.ac.cr ORCID: https://orcid.org/0000-0003-4977-2397 


\section{ABSTRACT}

Orcas are little studied cetaceans in the tropics, and their movements through Costa Rican waters have been scarcely addressed. They are animals of great ecological importance due to their role as top predators and documenting their presence in the country can be of great scientific and social importance, for coastal communities this information could represent a great opportunity from an ecotourism perspective. The objective of this research was to synthesize and systematize the existing reports regarding orcas in the country, using scientific publications, books and information derived from citizen reports. The information collected up to December 31, 2020, was considered. The sightings and the number of killer whales in the Costa Rican Pacific were divided regionally: 17 sightings and 56 individuals for the South Pacific, 7 sightings and 20 individuals for the North Pacific. 3 sightings and 19 individuals in the Central Pacific and 4 sightings and 10 individuals for Isla del Coco. There were 31 sightings and 105 individuals in total. No statistically significant differences were found in the occurrence of killer whales between the zones, although there is a greater number of reports in the South Pacific, especially in September. Studying the presence of orcas in the country, involving processes of environmental education and citizen science, can provide important tools for the formulation of public policies that allow an effective conservation of cetaceans and offer greater opportunities for growth for the ecotourism industry in the coasts.

Keywords: cetacean displacement, Citizen science, predatory habits, tropical marine mammal ecology, tropical killer whales

\section{INTRODUCCIÓN}

El estudio de los cetáceos marinos ha sido una actividad de gran importancia para las sociedades humanas debido a que permite comprender dinámicas ecosistémicas (Parsons et al. 2015). Se han efectuado varias investigaciones referentes a características ecológicas de los cetáceos residentes y visitantes en las aguas de nuestro país (Steiger et al. 1991; Wade \& Gerrodette, 1993; Acevedo \& Smultea, 1995; Acevedo \& Burkhart, 1998; May-Collado \& Morales-Ramírez, 2005; Rasmussen et al. 2007; Palacios-Alfaro, 2009; Rasmussen et al. 2012; Martínez-Fernández et al. 2011; May-Collado \& Forcada, 2012;
Martínez-Fernández et al. 2014; Acevedo et al. 2017), y la información devengada no solo ha permitido tener una mejor idea de la diversidad del grupo en el país, adicionalmente nos provee herramientas para comprender, de mejor manera, los ensamblajes tróficos existentes en nuestro territorio marítimo (May-Collado et al. 2018; Fourriére et al. 2019). Esto facilitó el desarrollo de una importante industria de observación de cetáceos, de forma predominante en el Pacífico Sur del país, y enriquecer el conocimiento sobre las orcas en el país, posiblemente, permitiría aumentar el interés del público general, $\mathrm{y}$, por consiguiente, atraer un mayor número de personas que deseen ver estos animales (Rodríguez-Fonseca \& 
Fischel-Quirós, 2007; Hoyt \& Iñíguez, 2008; Montero-Cordero \& Lobo, 2010). Esta actividad permite una distribución de recursos importante entre los miembros de las comunidades que participan en ella, al ser altamente descentralizada (Cisneros-Montemayor \& Sumaila, 2010) y de base comunitaria, al emplear personas en transporte, hospedaje, alimentación, tours y educación ambiental (Wilson \& Tisdell, 2003; Hoyt \& Iñ́guez, 2008). Si bien este tipo de actividad puede resultar nociva para los hábitos de los cetáceos, si no se toman precauciones, la misma está normalizada por un reglamento que es parte de la legislación nacional y que está basado en estrategias de mitigación puestas en práctica, que buscan garantizar la sostenibilidad de esta industria (May-Collado \& Wartzok, 2008). Diferentes ONG especializadas, con apoyo del SINAC/MINAE, ICT e INA han dado capacitaciones en distintos momentos sobre dicho reglamento tanto a las autoridades involucradas en su implementación como a los capitanes, guías y operadores de la actividad (Nel-Lo Andreu, 2008; Carvache-Franco et al. 2021).

Los cetáceos son de importancia ecológica como indicadores ambientales, ya que sus migraciones pueden ayudar a comprender el impacto del cambio climático y la salud de los ecosistemas que frecuentan. Sus patrones de movimiento y alimentación están ligados a factores como características geográficas, temperatura del agua, topografía del fondo marino y disponibilidad de determinadas presas. En general, por su posición en las redes tróficas marinas son bioindicadores clave en los ecosistemas marinos (Dahlheim \& White, 2010; Filatova et al. 2019). Durante la última década la conservación de los cetáceos en el nivel mundial ha recibido mucha atención, debido a su capacidad de fijación de carbono, así como importantes interacciones que presentan con el fitoplancton, en donde la actividad de cetáceos promueve la proliferación de estos microorganismos (Chami et al. 2019). Para nuestro país se ha informado un total de 31 especies de cetáceos, siendo el tercer grupo de mamíferos más diverso (Rodríguez-Herrera et al. 2014).

La región tiene faltantes de información sobre las visitas de especies depredadoras como Orcinus orca (Martínez-Fernández et al. 2011). Existen informes sobre la presencia de estos animales en el país (Rodríguez-Fonseca, 2001; Sáenz et al. 2004; May-Collado et al. 2005; Martínez-Fernández et al. 2011). En los últimos años han trascendido referencias de personas que logran fotografiarlos o incluso filmarlos (Castro, 2019; Cabezas, 2020; Jerez, 2020). Sin embargo, es poco lo que se sabe sobre ellos. Hay publicaciones de estos depredadores alimentándose de tiburones (Carcharhinidae) en nuestro territorio (Fertl, 1996). Se les ha observado cerca de la Isla del 
Coco alimentándose del tiburón tigre (Galeocerdo cuvier) (Herrera, 2014), pero se registran pocos informes de los hábitos alimentarios de aquellos que se acercan más a la costa, en regiones tropicales (Guerrero-Ruiz et al. 1998; Salinas \& Urbán, 2006). Al ser una especie de fácil identificación debido a sus características morfológicas, permite acercar a la población a procesos de educación ambiental y facilita la implementación de herramientas de ciencia ciudadana para recopilar datos que demandan de grandes esfuerzos de muestreo, tales como los desarrollados para realizar estudios sobre diversidad y observar patrones migratorios de aves (Dickinson et al. 2010; Cooper et al. 2014). El objetivo principal de esta investigación consistió en sintetizar la información existente, referente a las orcas en el país, mediante publicaciones científicas, libros e información derivada de mecanismos de sistematización basados en informes de ciudadanos. Parte del interés de realizar este trabajo es, contar con una base para poder establecer comparaciones futuras, para así, poder determinar, si en efecto, la frecuencia de avistamientos de estos animales a nuestras aguas está cambiando con el tiempo.

\section{MATERIALES Y MÉTODOS}

Se dividieron las regiones en Isla del Coco, Pacífico Norte, Pacífico
Central y Pacífico Sur (Fig. 1). Para realizar esta división se tomaron en cuenta características oceanográficas, geográficas y ecosistémicas, como el domo térmico en el Pacífico Norte (Tassi et al. 2018), la lejanía con respecto a la masa continental de la Isla del Coco (Arias et al. 2016), las características ecosistémicas particulares del Pacífico Sur (Cortés \& Quesada-Alpízar, 2006) y las características ecosistémicas del Pacífico Central, la cual presenta un mayor impacto que las anteriores debido a las perturbaciones antropogénicas (Sánchez-Gutiérrez et al. 2020).

Se omitió la región Caribe porque únicamente se encontró una publicación al respecto (Bolaños-Jiménez et al. 2014). Durante la primera etapa de investigación se realizó una exhaustiva revisión bibliográfica sobre todas las fuentes que se pudiesen encontrar acerca de las orcas en Costa Rica. La información se consultó en inglés y en español. Se consultaron libros y artículos científicos mediante las bases de datos JStor (https://www.jstor.org/) y Science Direct (https://www.sciencedirect.com/) utilizando las palabras clave Orcinus orca, killer whale, Costa Rica y Central América.

Se tomaron en cuenta los datos de un avistamiento de tres maneras: publicación científica, por formulario avistamiento y en redes sociales. Se trabajó con cada una de la siguiente manera: 


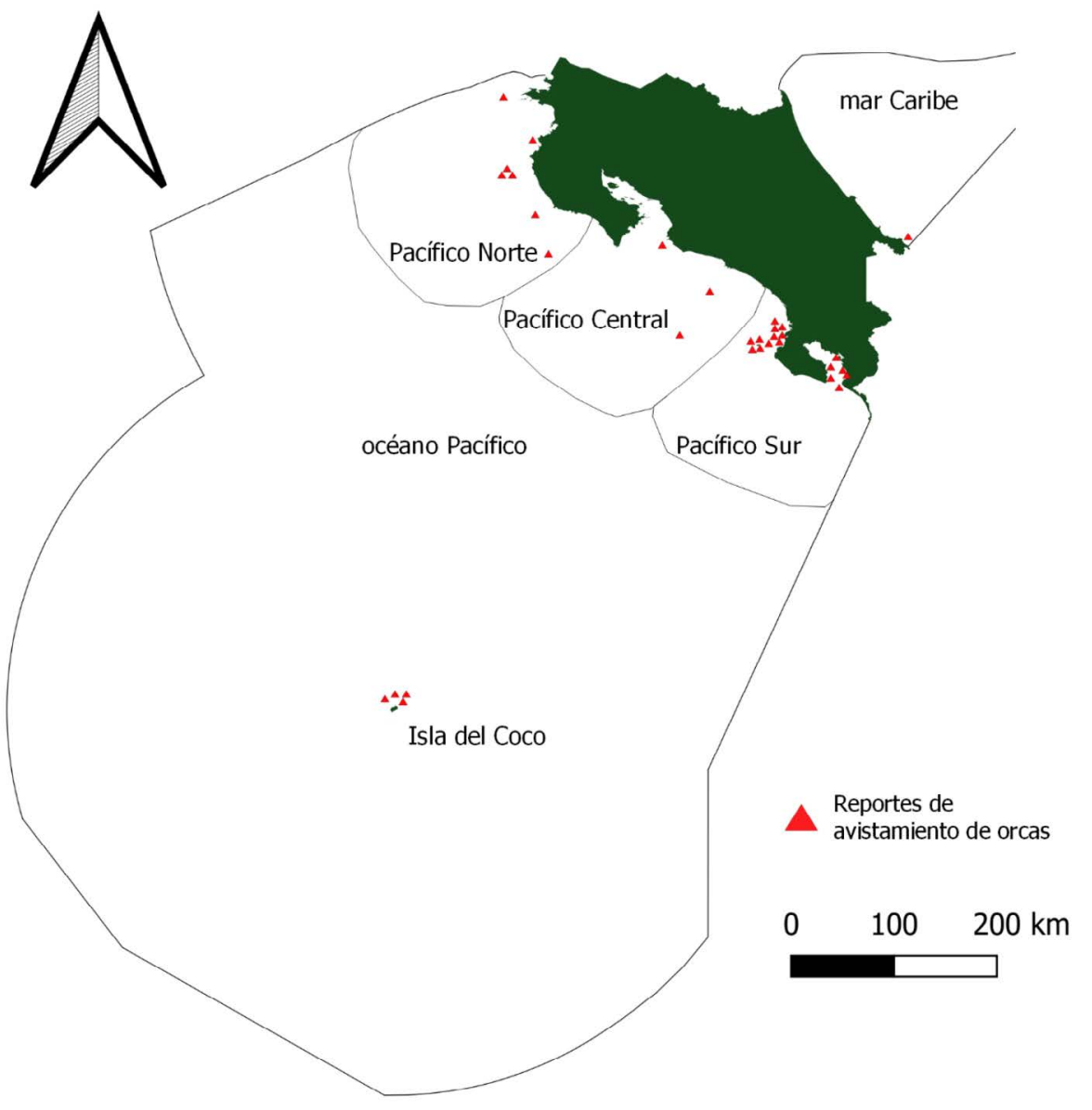

Fig 1. Croquis de aguas jurisdiccionales de Costa Rica con sitios en los que se reportaron observaciones de orcas

Fig 1. Diagram of the jurisdictional waters of Costa Rica with reported sightings of orcas

\section{Publicación científica:}

Se obtuvieron datos de avistamiento propiedad de Cascadia Research Collective (Olympia, Washington, EE. UU.), así como publicaciones de artículos científicos derivados de la búsqueda en las bases de datos antes mencionadas.

\section{Formulario:}

Se diseñó un formulario donde se consultaba por el sitio de avistamiento, tamaño de grupo y fotografías o videos como evidencia. Se entregó a operadores turísticos de las regiones Norte, Central y Sur, del Pacífico costarricense, en formato digital, entre el 
20 de setiembre y el 20 de octubre de 2020. Las respuestas a este formulario se recopilaron hasta el 31 de diciembre de 2020. Se realizaron entrevistas telefónicas y mediante videollamadas a 13 personas, 6 investigadores nacionales e internacionales, 2 trabajadores de la pesca, 2 del sector de transporte marítimo, 1 persona del Servicio Nacional de Guardacostas y 2 operadores turísticos. También se tomaron en cuenta 4 informes proporcionados por investigadores, pero que no cuentan con una publicación científica oficial, y, para efectos de análisis de datos, se separaron de las demás categorías debido a que se consideró que podrían resultar útiles, por separado, para estudios posteriores.

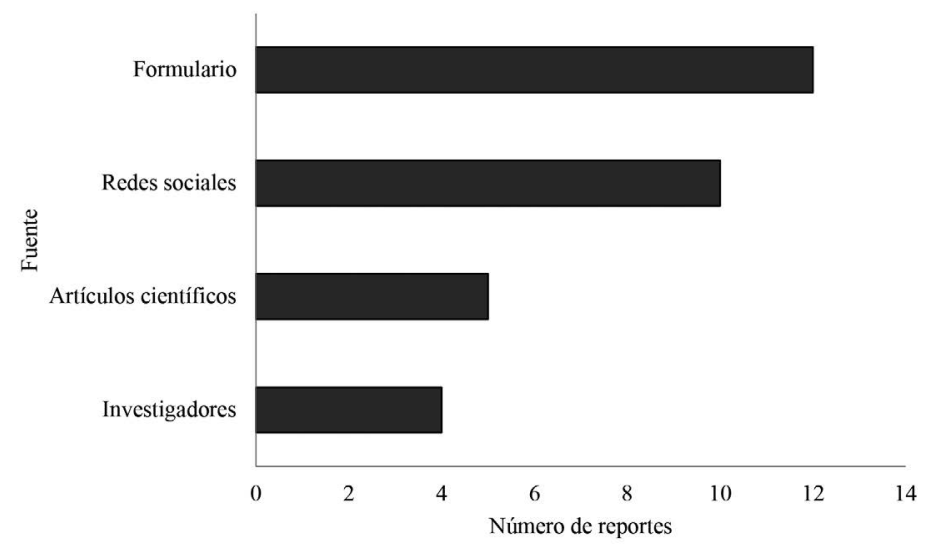

Fig 2. Cantidad de avistamientos de Orcinus orca en el Pacífico costarricense por cada una de las cuatro categorías de fuentes de información utilizadas para el período 1990-2020

Fig 2. Number of sightings of Orcinus orca spotted in the Costa Rican Pacific by each one of the four categories of sources of information used for the period 1990-2020

\section{Redes sociales:}

Se realizó una revisión de publicaciones en redes sociales (Facebook, Instagram y YouTube) que incluían fotografías y videos de orcas en el país, se contactó a las personas responsables del material. Se creó una base de datos para condensar la información derivada de artículos científicos, respuestas al formulario, información facilitada por investigadores y redes sociales.

\section{Análisis de datos}

Para determinar si existen diferencias entre el número de orcas observadas y el sitio de reporte se realizó un análisis de varianza (ANOVA), mediante la plataforma estadística RStudio (2020), con un nivel de significancia de 0.05 y con intervalos de confianza al $95 \%$. Se esperaba que existiera diferencia entre los sitios, por la mayor cantidad de reportes para la región Pacífico Sur. Se realizó un gráfico para comparar las fuentes de los reportes (Fig. 2). 


\section{RESULTADOS}

Se constituyó una base de datos para realizar el análisis de la información, a partir de las 12 respuestas al formulario, información obtenida de redes sociales, información facilitada por investigadores y artículos científicos entre 1990 y 2020 (Cuadro 1). La mayoría de los datos recopilados corresponden a respuestas al formulario, que obtuvo 12 , en las redes sociales se consiguieron

Cuadro 1. Base de datos con fechas de avistamiento y tamaño de grupos de Orcinus orca en el Pacífico costarricense entre 1990 y 2020

Table 1. Dates of sightings and sizes of groups of Orcinus orca in the Costa Rican Pacific between 1990 and 2020

\begin{tabular}{|c|c|c|c|c|}
\hline Mes & Año & Sitio & Tamaño de grupo & Origen del informe \\
\hline Mayo & 1992 & Pacífico Sur & 4 & Artículo científico \\
\hline Febrero & 1997 & Pacífico Sur & 1 & Artículo científico \\
\hline Febrero & 1997 & Pacífico Sur & 3 & Artículo científico \\
\hline Enero & 2003 & Pacífico Sur & 7 & Artículo científico \\
\hline Enero & 2006 & Pacífico Norte & 1 & Artículo científico \\
\hline Junio & 2008 & Pacífico Sur & 1 & Redes sociales \\
\hline Junio & 2010 & Pacífico Sur & 3 & Investigador \\
\hline Setiembre & 2012 & Isla del Coco & 2 & Redes sociales \\
\hline Setiembre & 2012 & Isla del Coco & 2 & Redes sociales \\
\hline Setiembre & 2012 & Pacífico Sur & 1 & Formulario \\
\hline Junio & 2013 & Pacífico Norte & 6 & Investigador \\
\hline Agosto & 2013 & Pacífico Sur & 1 & Investigador \\
\hline Setiembre & 2013 & Pacífico Sur & 1 & Formulario \\
\hline Setiembre & 2014 & Isla del Coco & 3 & Redes sociales \\
\hline Noviembre & 2016 & Pacífico Sur & 2 & Formulario \\
\hline Diciembre & 2016 & Pacífico Central & 3 & Redes sociales \\
\hline Marzo & 2017 & Pacífico Sur & 1 & Redes sociales \\
\hline Diciembre & 2017 & Pacífico Norte & 3 & Formulario \\
\hline Abril & 2018 & Pacífico Sur & 10 & Redes sociales \\
\hline Setiembre & 2018 & Pacífico Norte & 1 & Redes sociales \\
\hline Febrero & 2019 & Pacífico Sur & 3 & Formulario \\
\hline Agosto & 2019 & Pacífico Central & 7 & Formulario \\
\hline Setiembre & 2019 & Isla del Coco & 3 & Formulario \\
\hline Setiembre & 2019 & Pacífico Sur & 3 & Formulario \\
\hline Diciembre & 2019 & Pacífico Sur & 10 & Redes sociales \\
\hline Marzo & 2020 & Pacífico Central & 9 & Redes sociales \\
\hline Abril & 2020 & Pacífico Norte & 4 & Investigador \\
\hline Abril & 2020 & Pacífico Norte & 3 & Formulario \\
\hline Mayo & 2020 & Pacífico Sur & 3 & Formulario \\
\hline Junio & 2020 & Pacífico Sur & 2 & Formulario \\
\hline \multirow[t]{2}{*}{ Diciembre } & 2020 & Pacífico Norte & 2 & Formulario \\
\hline & & Total & 105 & \\
\hline
\end{tabular}


Cuadro 2. Distribución de los avistamientos de Orcinus orca en el Pacífico costarricense entre 1990 y 2020 en las regiones Norte, Central, Sur e Isla del Coco Table 2. Distribution of Orcinus orca sightings in the Costa Rican Pacific between 1990 and 2020 in the Northern, Central and Southern regions, and Cocos Island

\begin{tabular}{lccc}
\hline \multicolumn{1}{c}{ Región } & Avistamientos & Animales & Promedio de animales por avistamiento \\
\hline Norte & 7 & 20 & $2.9( \pm 1.3)$ \\
Central & 3 & 19 & $6.3( \pm 2.2)$ \\
Sur & 17 & 56 & $3.3( \pm 2.1)$ \\
Isla del Coco & 4 & 10 & $2.5( \pm 0.5)$ \\
\hline
\end{tabular}

10 reportes, 5 derivaron de artículos científicos y 4 fueron suministrados por investigadores (Fig. 2). En resumen, las respuestas válidas al formulario consistieron en videos, fotografías y mensajes que documentaban encuentros con orcas y podían verificarse mediante la información suministrada. Hubo 5 respuestas al formulario sobre Megaptera novaengliae y Tursiops truncatus, no tomadas en cuenta.

Se recopiló un total de 31 avistamientos. Se observaron 105 individuos en total. La región Pacífico Sur fue la que registró una mayor cantidad de avistamientos y un mayor número de orcas (Cuadro 2). La región Pacífico Central solo registró 3 avistamientos, pero constaron de grupos más grandes, por lo que registró más orcas que las regiones Pacífico Norte e Isla del Coco (Cuadro 2). En las regiones Pacífico Sur y Pacífico Norte existen informes de todos los meses, excepto julio y octubre. En el Pacífico Central existen informes en marzo, agosto y diciembre, mientras en la Isla del Coco solo se registraron en setiembre (Cuadro 1). Los años 2019 y 2020 fueron los que más informes registraron, con 5 cada uno, siendo el 2019 el año en el que se obtuvo un mayor número de orcas, con 26 (Cuadro 1). Dos personas de las 13 entrevistadas manifestaron haberlas visto alimentarse de atunes (Scombridae), jureles (Carangidae) y una tortuga (Cheloniidae); además, mencionaron que las orcas son vistas poco tiempo después de la llegada de las ballenas jorobadas.

Setiembre fue el mes que más avistamientos y número de orcas registró. Diciembre fue el segundo en cuanto a número de orcas, mientras que junio fue el segundo en cuanto a avistamientos. Ni julio ni octubre contaron con avistamientos (Cuadro 1). La actividad depredadora registrada incluyó el consumo de mantarrayas (Mobulidae) y en un vídeo se les pueden ver acosando a una cría de tiburón ballena (Rhyncodon typus). Para el Pacífico Central los avistamientos fueron escasos, y con distancias entre 20 y 80 millas náuticas 


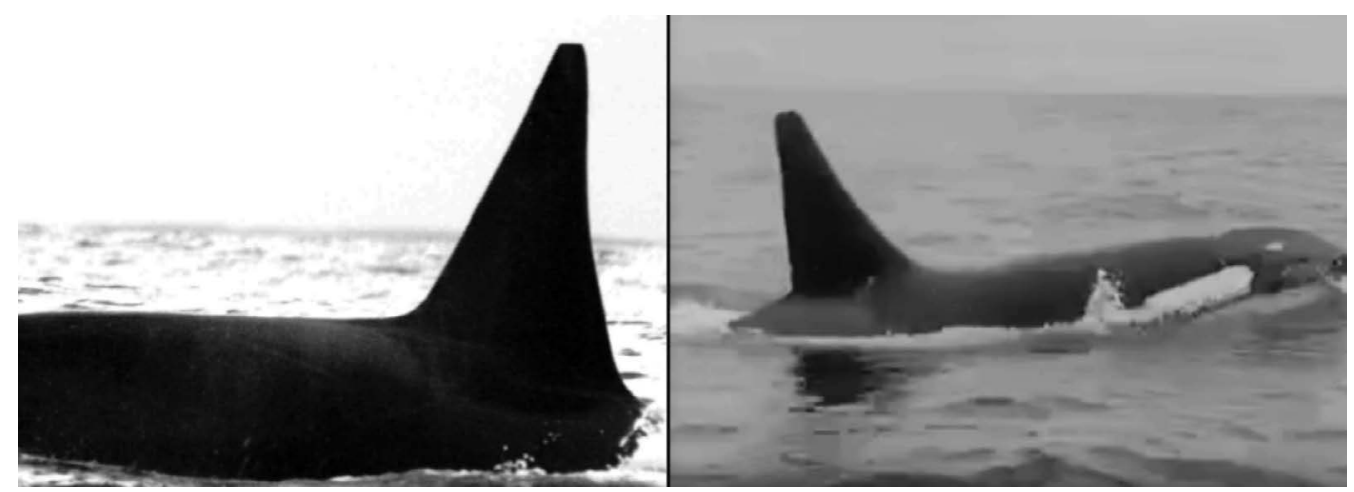

Fig 3. Comparación de imágenes de vista de la aleta dorsal vista desde el costado derecho del macho iwdentificado como E231 en el catálogo de orcas del Pacífico Tropical Oriental (izquierda), y macho observado en el Pacífico Sur en 2020 con gran similitud (derecha). Fotografía derecha propiedad de Sequeira Tours

Fig 3. Comparison of dorsal fin view from the right side of the male identified as E231 in the Orca catalog of the Eastern Tropical Pacific (left), and male observed in the South Pacific in 2020 with great similarity (right). Right photograph property of Sequeira Tours

de la costa. En el Pacífico Sur los entrevistados indicaron que las orcas pueden verse durante todo el año, pero siendo más probable a finales de agosto $\mathrm{y}$ principios de setiembre. En el Pacífico Sur se han visto cerca de la costa, y la zona que reporta más avistamientos está comprendida entre Bahía Drake y la Reserva Biológica Isla del Caño. Para esta zona, se identificó un macho con características muy similares (Fig. 3) a las reportadas en el catálogo de orcas del Pacífico Tropical Oriental, para el macho E231 (Olson \& Gerrodette, 2008). En el Parque Nacional Isla del Coco, se observaron grupos de orcas cerca de la isla, y, en una ocasión, se les contempló alimentándose del tiburón tigre (Galeocerdo cuvier). Todos los avistamientos en esta zona ocurrieron en setiembre.

Según la prueba ANOVA, no existe una diferencia significativa entre el número de orcas que se pueden encontrar entre los diferentes sitios analizados $(F=1.582 ; P>0.05)$. Las discrepancias entre la cantidad de observaciones entre sitios y la relativa similitud entre los promedios de animales, una vez que se pondera el error, podrían explicar por qué el resultado de la prueba estadística no fue significativo. A pesar de este resultado, los datos sí muestran una marcada diferencia en cuanto a la cantidad de avistamientos entre las regiones (Cuadro 2). 


\section{DISCUSIÓN}

Plantear el proyecto para recopilar avistamientos de orcas en el país y estructurar la toma de datos, de forma amigable, con el público, permitió utilizar una amplia diversidad de datos y de fuentes. Involucrar a personas no vinculadas a la academia en el proceso de recopilación de información, con fines científicos, facilita el trabajo con animales difíciles de encontrar en aguas tropicales como las orcas (dos Santos \& Bessa, 2019), aunque esto presente limitantes como que los informes sean esporádicos y que sea necesario verificarlos con vídeos o fotografías para evitar errores de identificación.

La falta de avistamientos en julio y octubre puede deberse al comportamiento migratorio de otros organismos como M. novaengliae (May-Collado et al. 2005). Estas ballenas, normalmente, no empiezan a llegar en grandes grupos al país hasta agosto, como parte de la migración de las poblaciones del hemisferio sur (Rasmussen et al. 2007). Las poblaciones del hemisferio norte llegan, en los primeros meses del año, a las cálidas aguas del Pacífico costarricense, evitando las condiciones del frío invierno en el hemisferio norte en esa época (Steiger et al. 1991).

Una posible explicación es que los desplazamientos de las orcas en la región se deben a la migración de la ballena jorobada $M$. novaengliae. Aunque no existen registros en el país de orcas cazándolas, se sabe que en otros sitios las depredan con frecuencia (Steiger et al. 2008). Con respecto a octubre, las condiciones, por lo general lluviosas, en nuestro país (Mendez et al. 2019) podrían influenciar la falta de observaciones, pues complicarían la navegación y visibilidad, pero también deben tomarse en cuenta los fenómenos migratorios propios de las ballenas, antes descritos.

Algunos videos en redes sociales hacen referencia al mismo evento, tomados por diferentes personas en las mismas embarcaciones. Para la investigación se tomó el reporte que primero se subió a la red social, en caso de existir varios (Cherson, 2014; Herrera, 2014; Power, 2014). Una ventaja de este tipo de fuente de información es, que permite verificar, desde distintas perspectivas, que en efecto ocurrió el encuentro con orcas en la fecha y sitio estipulados, y, además, es posible hacer un mejor conteo de los animales si se tienen diferentes perspectivas.

Para las 13 personas entrevistadas, estas observaciones son un fenómeno que, aunque infrecuente, no es nuevo, pues todos ellos confirmaron que, en los últimos 20 años, se han realizado múltiples observaciones de orcas en altamar e incluso cerca de la costa, pero que no se habían documentado apropiadamente. Conviene mencionar que el alto número de 
observaciones en el Pacífico Sur, en particular entre Bahía Drake y la Isla del Caño, puede tener una estrecha relación con las actividades turísticas de observación de cetáceos en la zona (Rodríguez-Fonseca \& Fischel-Quirós 2007; Hunt et al. 2015), y quizá, en las demás, no hay tantos avistamientos, simplemente porque existe un menor número de personas en el mar observando animales.

Otro grupo importante de observaciones ocurre en el golfo Dulce, clasificado en este trabajo dentro de la región Pacífico Sur, donde se les ha visto alimentarse de elasmobranquios y peces óseos (Fertl, 1996). Este comportamiento predatorio ya ha sido observado en otras poblaciones de orcas (Secchi \& Vaske, 1998; Samarra et al. 2018). La depredación de estos animales puede deberse a que son presas más abundantes en estas aguas, por lo que, depredadores oportunistas como las orcas podrían aprovecharlos. Además de los beneficios ecosistémicos que los grandes depredadores como las orcas nos proporcionan mediante la regulación de cascadas tróficas (Winnie Jr. \& Creel, 2017; Rao, 2018), pueden contribuir con el sustento económico de esta zona, al ser un grupo altamente carismático y de importancia ecoturística.

Aunque la cantidad de avistamientos es relativamente baja en cada sitio, es de gran utilidad segmentarla en las 4 zonas propuestas en este trabajo debido a que están sujetas a distintos tipos de presión antropogénica, características oceanográficas y fenómenos ambientales (Montero-Cordero \& Lobo, 2010; Tassi et al. 2018; Sánchez-Gutiérrez et al. 2020), que serán discutidos en cada caso. Este análisis, enfocado en zonas, permitirá establecer comparaciones futuras y buscará generar información valiosa a los investigadores y a las poblaciones de dichas localidades dedicadas al ecoturismo.

Para la región Pacífico Central hubo 3 avistamientos (Cuadro 1). La polución asociada a los focos de población humana (González-Fernández, 2021), en esta región, podría hacer que estos cetáceos no se observen con frecuencia, pues se sabe que tanto las aguas contaminadas como el excesivo ruido antropogénico puede resultar disruptivo para la comunicación de cetáceos similares, quienes tenderán a evitarlos (Montero-Cordero \& Lobo, 2010).

En el Pacífico Norte hubo 7 avistamientos (Cuadro 1), y se documentaron atacando mantarrayas y un tiburón ballena juvenil (Rhyncodon typus). Principalmente, fueron vistos cerca de las Islas Catalinas. En los videos que se recopilaron de esta zona, se observaron individuos exhibiendo comportamientos asociados a alarma, dando grandes saltos fuera del agua y acercándose a las embarcaciones. Las 3 personas entrevistadas, de esta zona, mencionaron que se podrían avistar orcas con mayor frecuencia a 
final y principio de año, coincidiendo con el fenómeno de enfriamiento asociado al sistema de afloramiento de Papagayo (Alfaro \& Cortés, 2012). La influencia de este fenómeno debe tomarse en cuenta para estudiar este grupo, pues crea condiciones de alta productividad (Álvarez et al. 2018), que permiten la conformación de complejas redes tróficas, las cuales favorecen la presencia estacional de muchos organismos que las orcas podrían depredar. Existe un reporte en la zona del domo térmico de Costa Rica (fuera de aguas territoriales), de un grupo de orcas interactuando para alimentarse de una cría de Balaenoptera musculus (Pitman et al. 2007).

La Isla del Coco es un Parque Nacional de enorme importancia para la biodiversidad marina gracias a las condiciones geológicas de corrientes y climáticas que posee, además de la distancia a la que está, de focos de población humana y la protección legal que posee (Claudino-Sales, 2019). En esta región se registraron 4 avistamientos, y la relativa cercanía de las Islas Galápagos hace pensar que, posiblemente, estos animales utilicen la ruta de migración entre Galápagos e Isla del Coco, como parte de sus rutas de alimentación y otras actividades, fungiendo como importantes reguladores, en las redes tróficas de ambos sitios, tal como se ha observado en elasmobranquios pelágicos (Nalesso et al. 2019; Bessudo et al. 2011).
A pesar que la literatura menciona la posibilidad de encontrar orcas en el Caribe (Sáenz et al. 2004), únicamente se encontró una publicación que confirma su presencia en el Caribe costarricense, cerca del Refugio Nacional de Vida Silvestre Gandoca-Manzanillo (Bolaños-Jiménez et al. 2014). Teniendo en cuenta la diversidad de cetáceos que se pueden encontrar en estas aguas (May-Collado, 2009), puede ser que pasaran desapercibidas debido a una menor cantidad de actividades ecoturísticas respecto al Pacífico. Recientes reportes de orcas en la costa Caribe de Colombia, cerca de la frontera con Panamá (Franco \& Delgadillo, 2021) indican que, tal vez con un mayor esfuerzo de muestreo, se pueda registrar, de manera más exacta, su presencia en el Caribe de Costa Rica.

En un vídeo del sector Pacífico Sur del país, del año 2020, se observó un macho (Fig. 3) con características muy similares al identificado como E231, reportado en el catálogo de orcas del Pacífico Tropical Oriental, cerca de la Isla Clipperton, en el año 2006 (Olson \& Gerrodette, 2008). El macho conocido como "Phantom" fue documentado realizando movimientos entre Perú, Ecuador, Costa Rica y México que coinciden con las temporadas de apareamiento de la ballena jorobada en ambos hemisferios (Pacheco et al. 2019). Existe otro caso documentado de un macho que fue fotografiado en Perú y en México, en ambos casos en 
compañía de otras orcas, con pocos años de diferencia entre ambos reportes (Guerrero-Ruiz et al. 2005). Estos datos sugieren que las orcas de la región pueden desplazarse largas distancias en la zona y estas pueden coincidir con las migraciones de las ballenas jorobadas.

Los grupos avistados en el país podrían estar compuestos por los mismos individuos, en años distintos, que podrían estar de paso en sus rutas de desplazamiento regional. Para determinarlo, con certeza, será necesario confeccionar un catálogo actualizado de orcas en la región y analizar registros de forma minuciosa, para realizar identificaciones. Este material permitiría establecer comparaciones con los registros de otras zonas y ayudar a estimar, de una forma más exacta, el tamaño de las poblaciones de Orcinus orca, su origen, la distancia que recorren y qué rutas utilizan. Esto último, podría estudiarse mediante el uso de rastreadores, pero debido a que los encuentros son infrecuentes, la identificación fotográfica podría proveer información confiable para gran cantidad de individuos, pues se observó que varios presentaban marcas y cicatrices útiles para identificación.

Las interacciones de depredación en el país parecen estar orientadas hacia elasmobranquios y a peces óseos (Fertl, 1996; Sáenz et al. 2004; Herrera, 2014). No existen reportes de orcas alimentándose de mamíferos en aguas del Pacífico costarricense, pero no puede descartarse, debido a que estos son presas habituales en otras latitudes, en particular la ballena jorobada (Whitehead \& Glass, 1985; Steiger et al. 2008). En otras regiones existe un estrecho vínculo entre las actividades que las orcas realizan y las características geográficas del sitio donde se encuentran, principalmente para optimizar el uso de su energía (Heimlich-Boran, 1988), por lo que las características oceanográficas de Costa Rica podrían influenciar su comportamiento. Los grupos de orcas que son avistados en aguas costarricenses parecen corresponder en características como tamaño de grupo y características predatorias con el de las orcas transeúntes, pero cabe mencionar que esta clasificación se ha hecho con base en observaciones en orcas de zonas templadas (Baird \& Dill, 1996), y, en el trópico, cabría analizar si la clasificación más conveniente es entre orcas oceánicas y costeras, pero para esto se necesitan estudios más minuciosos.

Conocer el estado de las poblaciones de orcas en nuestras aguas es de interés regional, pues permite acciones coordinadas de protección y brinda herramientas para formular políticas públicas orientadas a la preservación de este grupo de enorme importancia ecológica y económica. La coordinación entre instituciones públicas, empresa privada y ciudadanía es crucial para poder ampliar el conocimiento sobre este grupo y desarrollar las herramientas 
que nos permitan un manejo apropiado en el futuro. Las poblaciones costeras dedicadas al ecoturismo y a tours de observación de cetáceos podrían beneficiarse de la presencia de las orcas y de la difusión que se haga de estas hacia el público general, pues esto podría facilitar una mayor llegada de turistas e investigadores a estas zonas.

\section{CONCLUSIONES}

Con base en los datos analizados, la región más probable de encuentro con orcas es el Pacífico Sur, en setiembre. Hace falta un mayor esfuerzo de muestreo para contar con datos más exactos de esta especie en el país, y para conseguirlos es útil contar con un buen programa de investigación, por lo que procesos de educación ambiental son excelentes herramientas para motivar a más personas a documentar y reportar futuros avistamientos. Cabe destacar que en muchos de los videos se observaron turistas nadando muy cerca de grupos de orcas, incluso con crías. Es crucial aumentar los esfuerzos de educación ambiental para evitar potenciales situaciones de riesgo, tanto para las personas como para estos animales. En el Caribe existe un vacío significativo de información que debe subsanarse, pues preocupa que, debido al bajísimo número de informes existentes, se corre el riesgo de que no se tomen decisiones apropiadas para protegerlas como la especie clave que es dentro de sus ecosistemas. La región ofrece un excelente potencial ecoturístico que podría aprovecharse, de forma más eficiente, si se tuvieran datos más exactos sobre la presencia de orcas.

\section{AGRADECIMIENTOS}

Este trabajo fue llevado a cabo gracias a la colaboración de Cascadia Research Collective, Annie Douglas, Laura May-Collado, Juan José Alvarado, Nefertiti Rojas, Shawn Larkin, Wanner Cano, Marco Loáiciga, Minor Lara y Frank Garita, y las personas que tomaron de su tiempo para responder el formulario, aportando valiosa información, material bibliográfico, videos, fotografías y relatos que contribuyeron con el desarrollo de esta investigación. Se extiende un cálido agradecimiento a los revisores anónimos, pues sus aportes fueron de gran ayuda para mejorar este trabajo.

\section{REFERENCIAS}

Acevedo, A. \& Burkhart, S. (1998). Seasonal distribution of bottlenose (Tursiops truncatus) and pan-tropical spotted (Stenella attenuata) dolphins (Cetacea: Delphinidae) in Golfo Dulce, Costa Rica. Rev. Biol. Trop., 46 (Supl. 6), -91-101.

Acevedo, A., \& Smultea, M. A. (1995). First records of humpback whales including calves at Golfo Dulce and Isla del Coco, Costa Rica, suggesting 
geographical overlap of northern and southern hemisphere populations. Mar. Mammal. Sci., 11(4), 554-560.

Acevedo, J., Aguayo-Lobo, A., Allen, J., Botero-Acosta, N., Capella, J., Castro, C., ... \& Stevick, P. T. (2017). Migratory preferences of humpback whales between feeding and breeding grounds in the eastern South Pacific. Mar. Mammal. Sci., 33(4), 1035-1052. https://doi. org/10.1111/mms.12423

Alfaro, E. J. \& Cortés, J. (2012). Atmospheric forcing of cool subsurface water events in Bahía Culebra, Gulf of Papagayo, Costa Rica. Rev. Biol. Trop., 60(Supl. 2), 173-186.

Álvarez, S. L., Benavides-Morera, R., Brenes-Rodríguez, C. L. \& Saxon, D. B. (2018). Estructura del fitoplancton en las épocas seca y lluviosa en el golfo de Papagayo, Costa Rica. Rev. Cien. Mar. Cos., 10(2) 9-30. https://doi. org/10.15359/revmar.10-2.1

Arias, A., Pressey, R. L., Jones, R. E., Álvarez-Romero, J. G., \& Cinner, J. E. (2016). Optimizing enforcement and compliance in offshore marine protected areas: a case study from Cocos Island, Costa Rica. Oryx, 50(1), 18-26. https://doi.org/10.1017/ S0030605314000337

Baird, R. W. \& Dill, L. M. (1996). Ecological and social determinants of group size in transient killer whales. Beh. Ecol., 7(4), 408-416. https://doi.org/10.1093/ beheco/7.4.408

Bessudo, S., Soler, G. A., Klimley, P. A., Ketchum, J., Arauz, R., Hearn, A., ... \& Calmettes, B. (2011). Vertical and horizontal movements of the scalloped hammerhead shark (Sphyrna lewini) around Malpelo and Cocos Islands (Tropical Eastern Pacific) using satellite telemetry. Bol. Inves. Mar. Cost., 40, 91-106.
Bolaños-Jiménez, J., Mignucci-Giannoni, A. A., Blumenthal, J., Bogomolni, A., Casas, J. J., Henríquez, A., ... \& Rinaldi, R. (2014). Distribution, feeding habits and morphology of killer whales (Orcinus orca) in the Caribbean Sea. Mamm. Rev., 44(3-4), 177-189. https:// doi.org/10.1111/mam.12021

Cabezas, Y. (2020, diciembre, 28). Captan espectáculo de orcas en Guanacaste. $\{$ Video\} https://www.crhoy.com/ ambiente/video-captan-espectaculo-de-orcas-en-guanacaste/

Carvache-Franco, M., Carvache-Franco, W., Víquez-Paniagua, A. G., Carvache-Franco, O., \& Pérez-Orozco, A. (2021). Satisfaction and Its Relationship with Loyalty in Ecotourism: A Study in Costa Rica. J. Environ. Manag. Tour., $12(3$ (51)), 787-796. https:// doi.org/10.14505//jemt.12.3(51).18

Castro, K. (2019, agosto, 19). ¡Espectacular! Video capta avistamiento de orcas en Quepos. $\{$ Video\} https://www.crhoy. com/ambiente/espectacular-video-capta-avistamiento-de-orcas-en-quepos/

Chami, R., Cosimano, T., Fullenkamp, C. \& Oztosun, S. (2019). La solución de la Naturaleza al cambio climático: una estrategia para proteger a las ballenas puede limitar los gases de efecto invernadero y el calentamiento global. Finanzas y desarrollo: publicación trimestral del Fondo Monetario Internacional y del Banco Mundial, 56(4), 34-38.

Cherson, A. (2014, Setiembre, 26). Orcas at Isla del Coco, Costa Rica: 4. \{Video\} https://www.youtube.com/ watch? $\mathrm{v}=\mathrm{xKTc} 8$ Fxin-g

Cisneros-Montemayor, A. M. \& Sumaila, U. R. (2010). A global estimate of benefits from ecosystem-based marine recreation: potential impacts and implications for management. J. Bioec., 12(3), 245-268. https:// doi.org/10.1007/s10818-010-9092-7 
Claudino-Sales, V. (2019). Cocos Island National Park, Costa Rica. In Coastal World Heritage Sites (pp. 321326). Dordrecht, Springer. https://doi. org/10.1007/978-94-024-1528-5_47

Cooper, C. B., Shirk, J. \& Zuckerberg, B. (2014). The invisible prevalence of citizen science in global research: migratory birds and climate change. PloS one, 9(9), e106508. https://doi. org/10.1371/journal.pone.0106508

Cortés, J., \& Quesada-Alpízar, M. A. (2006). Los ecosistemas marinos del Pacífico sur de Costa Rica: estado del conocimiento y perspectivas de manejo. Rev. Biol. Trop., 54(Suppl. 1), 101-145.

Dahlheim, M. E. \& White, P. A. (2010). Ecological aspects of transient killer whales Orcinus orca as predators in southeastern Alaska. Wildlife Biol., 16(3), 308322. https://doi.org/10.2981/09-075

Dickinson, J. L., Zuckerberg, B. \& Bonter, D. N. (2010). Citizen science as an ecological research tool: challenges and benefits. Annu. Rev. Ecol. Evol. Syst., 41, 149-172. https://doi.org/10.1146/ annurev-ecolsys-102209-144636

dos Santos, R., \& Bessa, E. (2019). Dolphin conservation can profit from tourism and Citizen science. Environ. Dev., 32, 100467. https://doi.org/10.1016/j. envdev.2019.100467

Fertl, D. (1996). Reporte de ballenas asesinas (Orcinus orca) alimentándose de un tiburón de la familia carcharhinidae. Mar. Mamm. Sci., 4, 606-611.

Filatova, O. A., Shpak, O. V., Ivkovich, T. V., Volkova, E. V., Fedutin, I. D., Ovsyanikova, E. N., ... \& Hoyt, E. (2019). Large-scale habitat segregation of fish-eating and mammal-eating killer whales (Orcinus orca) in the western North Pacific. Polar Biol., 42(5), 931-941. https://doi. org/10.1007/s00300-019-02484-6
Fourriére, M., Alvarado, J. J., Cortés, J., Taylor, M. H., Ayala-Bocos, A., Azofeifa-Solano, J. C., ... \& Wolff, M. (2019). Energy flow structure and role of keystone groups in shallow water environments in Isla del Coco, Costa Rica, Eastern Tropical Pacific. Ecol. Model., 396, 74-85. https://doi.org/10.1016/j.ecolmodel.2019.01.004

Franco, L. \& Delgadillo, O. (2021). A new sighting of killer whale, Orcinus orca, in Caribbean inshore waters off Colombia. Cienc. Mar., 47(1), 61-70. https:// doi.org/10.7773/cm.v47i1.3129

González-Fernández, A., Symonds, E. M., Gallard-Gongora, J. F., Mull, B., Lukasik, J. O., Navarro, P. R., ... \& Harwood, V. J. (2021). Relationships among microbial indicators of fecal pollution, microbial source tracking markers, and pathogens in Costa Rican coastal waters. Water Res., 188, 116507. https:// doi.org/10.1016/j.watres.2020.116507

Guerrero-Ruiz, M., García-Godos, I. \& Urbán, J. (2005). Photographic match of a killer whale (Orcinus orca) between Peruvian and Mexican waters. Aquat. Mamm., 31(4), 438-441. https://doi. org/10.1578/AM.31.4.2005.438

Guerrero-Ruiz, M., Gendron, D. \& Urbán, J. (1998). Distribution, movements, and communities of killer whales (Orcinus orca) in the Gulf of California, Mexico. Rep. Int. Whal. Comm., 48, 537-543.

Heimlich-Boran, J. R. (1988). Behavioral ecology of killer whales (Orcinus orca) in the Pacific Northwest. Can. J. Zool., 66(3), 565-578.

Herrera, M. (2014, setiembre, 11). Video muestra orcas cazando un tiburón tigre en la Isla del Coco. \{Video\} https://www. nacion.com/ciencia/medio-ambiente/ video-muestra-orcas-cazando-un-tiburon-tigre-en-la-isla-del-coco/ROZBM7S6U5BI3OFXHHEB6FVRK4/ story/ 
Hoyt, E. \& Iñíguez, M. (2008). The state of whale watching in Latin America. EE. UU. The Whale and Dolphin Conservation Society.

Hunt, C. A., Durham, W. H., Driscoll, L. \& Honey, M. (2015). Can ecotourism deliver real economic, social, and environmental benefits? A study of the Osa Península, Costa Rica. J. Sust. Tour., 23(3), 339-357. https://doi.org/10.108 $0 / 09669582.2014 .965176$

Jerez, M. (2020, mayo, 2). ¿Efecto pandemia? Pescador se topa con hermosas ballenas orca en Nosara. \{Video\} https:// amprensa.com/2020/05/video-efectopandemia-pescador-se-topa-conhermosas-ballenas-orca-en-nosara/

Martínez-Fernández, D., Montero-Cordero, A. \& May-Collado, L. (2011). Cetáceos de las aguas costeras del Pacífico norte y sur de Costa Rica. Rev. Biol. Trop., 59(1), 283-290.

Martínez-Fernández, D., Montero-Cordero, A., \& Palacios-Alfaro, D. (2014). Áreas de congregación de cetáceos en el Pacífico norte de Costa Rica: recomendaciones para la gestión del recurso. Rev. Biol. Trop., 62(4), 99-108.

May-Collado, L. (2009). Marine mammals. In I. Wehrtmann \& J. Cortés (Eds.), Marine Biodiversity of Costa Rica, Central America (pp. 479-495). Berlin: Springer.

May-Collado, L., Amador-Caballero, M., Casas, J. J., Gamboa-Poveda, M. P., Garita-Alpízar, F., Gerrodette, T., ... \& Pérez, B. (2018). In M. Rossi-Santos \& C. Frinkl (Eds.), Ecology and conservation of cetaceans in Costa Rica and Panama (pp. 293-319). Germany: Springer.

May-Collado, L., Gerrodette, T., Calambokidis, J., Rasmussen, K. \& Sereg, I. (2005). Patrones de la distribución de avistamiento de cetáceos en la zona económica exclusiva del Pacífico de Costa Rica basados en datos colectados entre 1979 y 2001. Rev. Biol. Trop., 53(1-2), 249-263.

May-Collado, L. J. \& Forcada, J. (2012). Small-scale estimation of relative abundance for the coastal spotted dolphins (Stenella attenuata) in Costa Rica: the effect of habitat and seasonality. Rev. Biol. Trop., 60, 133-142.

May-Collado, L., \& Morales Ramírez, A. (2005). Presencia y patrones de comportamiento del delfín manchado costero, Stenella attenuata (Cetacea: Delphinidae) en el Golfo de Papagayo, Costa Rica. Rev. Biol. Trop., 53(1-2), 265-276.

May-Collado, L. J. \& Wartzok, D. (2008). A comparison of bottlenose dolphin whistles in the Atlantic Ocean: factors promoting whistle variation. J. Mammal., 89(5), 1229-1240. https://doi. org/10.1644/07-MAMM-A-310.1

Mendez, M., Calvo-Valverde, L. A., Maathuis, B. \& Alvarado-Gamboa, L. F. (2019). Generation of monthly precipitation climatologies for Costa Rica using irregular rain-gauge observational networks. Water, 11(1), 70. https://doi. org/10.3390/w11010070

Montero-Cordero, A. \& Lobo, J. (2010). Effect of tourist vessels on the behaviour of the pantropical spotted dolphin, Stenella attenuata, in Drake Bay and Caño Island, Costa Rica. J. Cet. R. Manag., 11(3), 285-291.

Nalesso, E., Hearn, A., Sosa-Nishizaki, O., Steiner, T., Antoniou, A., Reid, A., ... \& Arauz, R. (2019). Movements of scalloped hammerhead sharks (Sphyrna lewini) at Cocos Island, Costa Rica and between oceanic islands in the Eastern Tropical Pacific. PloS one, 14(3), e0213741. https://doi.org/10.1371/ journal.pone.0213741 
Nel-Lo Andreu, M. (2008). Organización y características del turismo rural comunitario en Costa Rica/Structure and characteristics of community-based rural tourism in Costa Rica. An. Geog. Univ. Complutense, 12(3), 787-696.

Olson, P. A. \& Gerrodette, T. (2008). Killer whales of the Eastern Tropical Pacific: a catalog of photo-identified individuals. EE. UU.: Departamento de Comercio.

Pacheco, A. S., Castro, C., Carnero-Huaman, R., Villagra, D., Pinilla, S., Denkinger, J., ... \& Urbán, J. (2019). Short Note Sightings of an Adult Male Killer Whale Match. Aquat. Mamm., 45(3), 320-326.

Palacios-Alfaro, J. D. (2009). First record of the dwarf sperm whale (Kogia sima) in Caribbean waters of Costa Rica. Primer registro del cachalote enano (Kogia sima) en aguas caribeñas de Costa Rica. Lat. Am. J. Aquat. Mamm., 7(1/2), 103. https://doi.org/10.5597/ lajam00145

Parsons, E. C. M., Baulch, S., Bechshoft, T., Bellazzi, G., Bouchet, P., Cosentino, A. M., ... \& Sutherland, W. J. (2015). Key research questions of global importance for cetacean conservation. Endang. Species Res., 27(2), 113-118. https:// doi.org/10.3354/esr00655

Pitman, R. L., Fearnbach, H., Leduc, R., Gilpatrick Jr, J. W. \& Ford, J. K. (2007). 151 Killer whales preying on a blue whale calf on the Costa Rica Dome: genetics, morphometrics, vocalizations and composition of the group. J. Cetacean Res. Manage, 9(2), 151-157.

Power, C. (2014, November, 14). Killer Whales Hunting a Tiger Shark. \{Video\} https://www.youtube.com/ watch? $=$ P-oO_JIZ8SU

Rao, T. R. (2018). Trophic Cascades. Res., 23(11), 1205-1213.
Rasmussen, K., Calambokidis, J., \& Steiger, G. H. (2012). Distribution and migratory destinations of humpback whales off the Pacific coast of Central America during the boreal winters of 1996-2003. Mar. Mamm. Sci., 28(3), E267-E279. https://doi. org/10.1111/j.1748-7692.2011.00529.x

Rasmussen, K., Palacios, D. M., Calambokidis, J., Saborío, M. T., Dalla Rosa, L., Secchi, E. R., ... \& Stone, G. S. (2007). Southern Hemisphere humpback whales wintering off Central America: insights from water temperature into the longest mammalian migration. Biol. Letters, 3(3), 302-305. https://doi. org/10.1098/rsbl.2007.0067

Rodríguez-Fonseca, J. (2001). Diversidad y distribución de los cetáceos de Costa Rica (Cetacea: Delphinidae, Physeteridae, Ziphiidae y Balaenopteridae). Rev. Biol. Trop. 49(2), 135-143.

Rodríguez-Fonseca, J. \& Fischel-Quirós. A. (2007). Impacto socioeconómico del turismo de observación de Cetáceos en Costa Rica. Informe Técnico FP04-07. Costa Rica. PROMAR-WSPA. Fundación Promar.

Rodríguez-Herrera, B., Ramírez-Fernández, J. D., Villalobos-Chaves, D., \& Sánchez, R. (2014). Actualización de la lista de especies de mamíferos vivientes de Costa Rica. Mast. Neotr., 21(2), 275-289.

RStudio Team. (2020). RStudio: Integrated Development for $R$. RStudio, PBC, EE. UU. http://www.rstudio.com/

Sáenz, J. C., Wong, G., Carrillo, E., Suárez, A., Zeledón, F., Collado, L. M. \& Iñíguez, M. (2004). Ballenas y delfines de América Central / Whales and Dolphins of Central America. Costa Rica: Editorial INBio. 
Salinas, M. \& Urbán, J. (2006). First mass stranding of killer whales (Orcinus orca) in the Gulf of California, Mexico. Aquat. Mamm., 32(3), 265-272.

Samarra, F. I., Bassoi, M., Béesau, J., Elíasdóttir, M. Ó., Gunnarsson, K., Mrusczok, M. T., ... \& Víkingsson, G. A. (2018). Prey of killer whales (Orcinus orca) in Iceland. PloS one, 13(12), e207287. https:/doi. org/10.1371/journal.pone.0207287

Sánchez-Gutiérrez, R., Mena-Rivera, L., Sánchez-Murillo, R., Fonseca-Sánchez, A., \& Madrigal-Solís, H. (2020). Hydrogeochemical baseline in a human-altered landscape of the central Pacific coast of Costa Rica. Environ. Geochem. H., 42(9), 2685-2701. https:// doi.org/10.1007/s10653-019-00501-5

Secchi, E. \& Vaske, T. (1998). Killer whale (Orcinus orca) sightings and depredation on tuna and swordfish longline catches in southern Brazil. Aquat. Mamm., 24, 117-122.

Steiger, G. H., Calambokidis, J., Sears, R., Balcomb, K. C. \& Cubbage, J. C. (1991). Movement of humpback whales between California and Costa Rica. Mar. Mammal Sci., 7(3), 306-310. https://doi.or$\mathrm{g} / 10.1111 / \mathrm{j} .1748-7692.1991 . t b 00105 . x$

Steiger, G. H., Calambokidis, J., Straley, J. M., Herman, L. M., Cerchio, S., Salden, D. R., ... \& Gabriele, C. M. (2008).
Geographic variation in killer whale attacks on humpback whales in the North Pacific: implications for predation pressure. End. Sp. Res., 4(3), 247-256. https://doi.org/10.3354/esr00078

Tassi, F., Vaselli, O., Bini, G., Capecchiacci, F., De Moor, J. M., Pecoraino, G., \& Venturi, S. (2018). The geothermal resource in the Guanacaste region (Costa Rica): new hints from the geochemistry of naturally discharging fluids. Front. Earth Sci., 6, 69. https://doi.org/10.3389/ feart.2018.00069

Wade, P. R. \& Gerrodette, T. (1993). Estimates of cetacean abundance and distribution in the eastern tropical Pacific Rep. Int. Whal., 43(477-493).

Whitehead, H. \& Glass, C. (1985). Orcas (killer whales) attack humpback whales. J. Mamm., 66(1), 183-185. https://doi. org/10.2307/1380982

Wilson, C. \& Tisdell, C. (2003). Conservación y beneficios económicos en turismo basado en vida silvestre marina: tortugas y ballenas como casos de estudio. Hum. Dimens. Wildl., 8(1), 49-58.

Winnie Jr, J. \& Creel, S. (2017). The many effects of carnivores on their prey and their implications for trophic cascades, and ecosystem structure and function. Food Webs, 12, 88-94. https://doi.org/10.1016/j.fooweb.2016.09.002 
